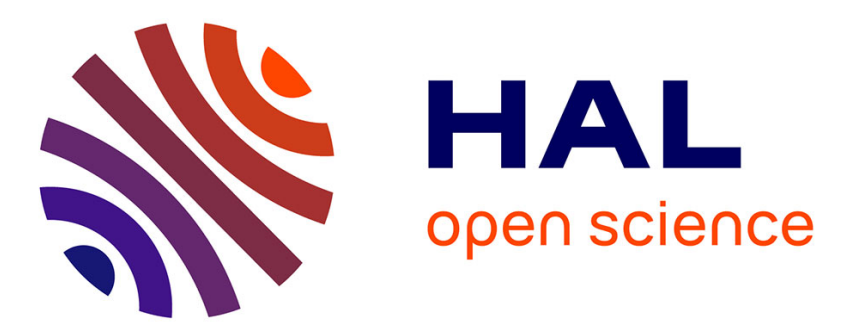

\title{
Weighted Homogeneity for Time-Delay Systems: Finite-Time and Independent of Delay Stability
}

Denis Efimov, Andrey Polyakov, Wilfrid Perruquetti, Jean-Pierre Richard

\section{To cite this version:}

Denis Efimov, Andrey Polyakov, Wilfrid Perruquetti, Jean-Pierre Richard. Weighted Homogeneity for Time-Delay Systems: Finite-Time and Independent of Delay Stability. IEEE Transactions on Automatic Control, 2016, 61, pp.210-215. 10.1109/TAC.2015.2427671 . hal-01145321v2

\section{HAL Id: hal-01145321 \\ https://inria.hal.science/hal-01145321v2}

Submitted on 15 May 2015

HAL is a multi-disciplinary open access archive for the deposit and dissemination of scientific research documents, whether they are published or not. The documents may come from teaching and research institutions in France or abroad, or from public or private research centers.
L'archive ouverte pluridisciplinaire HAL, est destinée au dépôt et à la diffusion de documents scientifiques de niveau recherche, publiés ou non, émanant des établissements d'enseignement et de recherche français ou étrangers, des laboratoires publics ou privés. 


\title{
Weighted Homogeneity for Time-Delay Systems: Finite-Time and Independent of Delay Stability
}

\author{
Efimov D., Polyakov A., Perruquetti W., Richard J.-P.
}

\begin{abstract}
Global delay independent stability is analyzed for nonlinear time-delay systems by applying homogeneity theory. It is shown that finite-time stability can be encountered in this class of systems under uniformity of the convergence time with respect to delay. Some additional tools for stability analysis of time-delay systems using homogeneity are also presented: in particular, it is shown that if a time-delay system is homogeneous with nonzero degree and it is globally asymptotically stable for some delay, then this property is preserved for any delay value, which is known as the independent of delay (IOD) stability.
\end{abstract}

\section{INTRODUCTION}

The theory of homogeneous dynamical systems has been well developed for continuous time-invariant differential equations [1], [2], [3], [4]. The main feature of a homogeneous nonlinear system is that its local behavior is the same as the global one (local attractiveness implies global asymptotic stability, for example). In addition, the homogeneous stable/unstable systems admit homogeneous Lyapunov functions [4], [5], [6]. Since the subclass of nonlinear systems with a global symmetry is rather narrow, the concept of local homogeneity has been introduced [4], [7], [6]. Then, a local homogeneous approximation of nonlinear system can be computed representing locally the original system behavior. The advantage in this case is that the approximating dynamics is globally homogeneous and the equation complexity is decreased.

The dynamical systems subjected by a time-delay, whose models are represented by functional differential equations, find their applications in many areas of science and technology [8], [9]. Analysis of delay influence on the system stability is critical for many natural and human-developed systems [10], [11], [12]. Despite of variety of applications, most of them deal with linear time-delay models, which is originated by complexity of stability analysis for time-delay systems in general (design of a Lyapunov-Krasovskii functional or a Lyapunov-Razumikhin function is a complex problem), and that constructive and computationally tractable conditions exist for linear systems only [13], [14].

An attempt to apply the homogeneity theory for nonlinear functional differential equations in order to simplify their stability analysis has been performed in [15], [16]. In those

The authors are with Non-A team @ Inria, Parc Scientifique de la Haute Borne, 40 av. Halley, 59650 Villeneuve d'Ascq, France and CRIStAL (UMRCNRS 9189), Ecole Centrale de Lille, BP 48, Cité Scientifique, 59651 Villeneuve-d'Ascq, France.

D. Efimov is with Department of Control Systems and Informatics, ITMO University, 49 Kronverkskiy av., 197101 Saint Petersburg, Russia.

This work was supported in part by the Government of Russian Federation (Grant 074-U01) and the Ministry of Education and Science of Russian Federation (Project 14.Z50.31.0031) works an extension of the homogeneity theory for time-delay systems has been proposed. Applications of the conventional homogeneity framework for analysis of time-delay systems (considering delay as a kind of perturbation, for instance) have been carried out even earlier in [17], [18], [19], [20], [21].

Establishing stability properties, it is also important to qualify the rate of convergence in the system: exponential, asymptotic, finite-time or fixed-time [22], [23], [24], [25], [26]. Frequently, the homogeneity theory is used to establish finite-time or fixed-time stability [2], [7], [26]: for example, if a system (without delay) is globally asymptotically stable and homogeneous of negative degree, then it is finite-time stable. There is a recent interest to analysis of finite-time stability behavior for time-delay systems [27], [28], [29], and the objective of the present paper is to show how the homogeneity theory extension proposed in [15], [16] can be used for analysis of finite-time stability for functional differential equations with nonzero degree of homogeneity.

The outline is as follows. The preliminary definitions and the homogeneity for time-delay systems are given in Section 2 . The scaling property of solutions for some class of homogeneous time-delay systems is investigated in Section 3, where the conditions of IOD stability are established. The conditions of finite-time stability are also given in Section 3. An example is considered in Section 4.

\section{PRELIMINARIES}

Consider an autonomous functional differential equation of retarded type [12]:

$$
d x(t) / d t=f\left(x_{t}\right), t \geq 0
$$

where $x \in \mathbb{R}^{n}$ and $x_{t} \in C_{[-\tau, 0]}$ is the state function, $x_{t}(s)=x(t+s),-\tau \leq s \leq 0$ (we denote by $C_{[-\tau, 0]}$ the Banach space of continuous functions $\phi:[-\tau, 0] \rightarrow \mathbb{R}^{n}$ with the uniform norm $\|\phi\|=\sup _{-\tau<\varsigma<0}|\phi(\varsigma)|$, where $|\cdot|$ is the standard Euclidean norm); $f: C_{[-\tau, 0]} \rightarrow \mathbb{R}^{n}$ ensures existence and uniqueness of solutions in forward time, $f(0)=0$. The representation (1) includes pointwise or distributed timedelay systems. We assume that solutions of the system (1) satisfy the initial functional condition $x_{0} \in C_{[-\tau, 0]}$. It is known from the theory of functional differential equations [12] that under the above assumptions the system (1) has a unique solution $x\left(t, x_{0}\right)$ satisfying the initial condition $x_{0}$ and $x_{t, x_{0}}(s)=x\left(t+s, x_{0}\right)$ for $-\tau \leq s \leq 0$, which is defined on some finite time interval $[-\tau, T$ ) (we will use the notation $x(t)$ to reference $x\left(t, x_{0}\right)$ if the origin of $x_{0}$ is clear from the context). 
The upper right-hand Dini derivative of a locally Lipschitz continuous functional $V: C_{[-\tau, 0]} \rightarrow \mathbb{R}_{+}$along the system (1) solutions is defined as follows for any $\phi \in C_{[-\tau, 0]}$ :

$$
D^{+} V(\phi)=\lim \sup _{h \rightarrow 0^{+}} \frac{1}{h}\left[V\left(\phi_{h}\right)-V(\phi)\right],
$$

where $\phi_{h} \in C_{[-\tau, 0]}$ for $0<h<\tau$ is given by

$$
\phi_{h}= \begin{cases}\phi(\theta+h), & \theta \in[-\tau,-h) \\ \phi(0)+f(\phi)(\theta+h), & \theta \in[-h, 0] .\end{cases}
$$

For a locally Lipschitz continuous function $V: \mathbb{R}^{n} \rightarrow \mathbb{R}_{+}$the upper directional Dini derivative is defined as follows:

$D^{+} V\left[x_{t}(0)\right] f\left(x_{t}\right)=\lim _{h \rightarrow 0^{+}} \sup \frac{V\left[x_{t}(0)+h f\left(x_{t}\right)\right]-V\left[x_{t}(0)\right]}{h}$.

A continuous function $\sigma: \mathbb{R}_{+} \rightarrow \mathbb{R}_{+}$belongs to class $\mathcal{K}$ if it is strictly increasing and $\sigma(0)=0$; it belongs to class $\mathcal{K}_{\infty}$ if it is also radially unbounded. A continuous function $\beta: \mathbb{R}_{+} \times \mathbb{R}_{+} \rightarrow \mathbb{R}_{+}$belongs to class $\mathcal{K} \mathcal{L}$ if $\beta(\cdot, r) \in \mathcal{K}$ and $\beta(r, \cdot)$ is decreasing to zero for any fixed $r \in \mathbb{R}_{+}$. The symbol $\overline{1, m}$ is used to denote a sequence of integers $1, \ldots, m$.

\section{A. Stability definitions}

Let $\Omega$ be a neighborhood of the origin in $C_{[-\tau, 0]}$.

Definition 1. [28] The system (1) is said to be

(a) stable at the origin into $\Omega$ if there is $\sigma \in \mathcal{K}$ such that for any $x_{0} \in \Omega,\left|x\left(t, x_{0}\right)\right| \leq \sigma\left(\| x_{0}||\right)$ for all $t \geq 0$;

(b) asymptotically stable at the origin into $\Omega$ if it is stable into $\Omega$ and $\lim _{t \rightarrow+\infty}\left|x\left(t, x_{0}\right)\right|=0$ for any $x_{0} \in \Omega$;

(c) finite-time stable at the origin into $\Omega$ if it is stable into $\Omega$ and for any $x_{0} \in \Omega$ there exists $0 \leq T^{x_{0}}<+\infty$ such that $x\left(t, x_{0}\right)=0$ for all $t \geq T^{x_{0}}$. The functional $T_{0}\left(x_{0}\right)=$ $\inf \left\{T^{x_{0}} \geq 0: x\left(t, x_{0}\right)=0 \forall t \geq T^{x_{0}}\right\}$ is called the settling time of the system (1).

If $\Omega=C_{[-\tau, 0]}$, then the corresponding properties are called global stability/asymptotic stability/finite-time stability.

\section{B. Homogeneity}

For any $r_{i}>0, i=\overline{1, n}$ and $\lambda>0$, define the dilation matrix $\Lambda_{r}(\lambda)=\operatorname{diag}\left\{\lambda^{r_{i}}\right\}_{i=1}^{n}$ and the vector of weights $r=$ $\left[r_{1}, \ldots, r_{n}\right]^{T}$.

For any $r_{i}>0, i=\overline{1, n}$ and $x \in \mathbb{R}^{n}$ the homogeneous norm can be defined as follows

$$
|x|_{r}=\left(\sum_{i=1}^{n}\left|x_{i}\right|^{\rho / r_{i}}\right)^{1 / \rho}, \rho \geq \max _{1 \leq i \leq n} r_{i} .
$$

For all $x \in \mathbb{R}^{n}$, its Euclidean norm $|x|$ is related with the homogeneous one:

$$
\underline{\sigma}_{r}\left(|x|_{r}\right) \leq|x| \leq \bar{\sigma}_{r}\left(|x|_{r}\right)
$$

for some $\underline{\sigma}_{r}, \bar{\sigma}_{r} \in \mathcal{K}_{\infty}$. The homogeneous norm has an important property that is $\left|\Lambda_{r}(\lambda) x\right|_{r}=\lambda|x|_{r}$ for all $x \in \mathbb{R}^{n}$. Define $\mathbb{S}_{r}=\left\{x \in \mathbb{R}^{n}:|x|_{r}=1\right\}$.
For any $r_{i}>0, i=\overline{1, n}$ and $\phi \in C_{[-\tau, 0]}$ the homogeneous norm can be defined as follows

$$
\|\phi\|_{r}=\left(\sum_{i=1}^{n}\left\|\phi_{i}\right\|^{\rho / r_{i}}\right)^{1 / \rho}, \rho \geq \max _{1 \leq i \leq n} r_{i} .
$$

There exist two functions $\underline{\rho}_{r}, \bar{\rho}_{r} \in \mathcal{K}_{\infty}$ such that for all $\phi \in$ $C_{[-\tau, 0]}[16]:$

$$
\underline{\rho}_{r}\left(\|\phi\|_{r}\right) \leq\|\phi\| \leq \bar{\rho}_{r}\left(\|\phi\|_{r}\right) .
$$

The homogeneous norm in the Banach space has the same important property that $\left\|\Lambda_{r}(\lambda) \phi\right\|_{r}=\lambda\|\phi\|_{r}$ for all $\phi \in C_{[-\tau, 0]}$. In $C_{[-\tau, 0]}$ the corresponding unit sphere $\mathcal{S}_{r}=\left\{\phi \in C_{[-\tau, 0]}\right.$ : $\left.\|\phi\|_{r}=1\right\}$. Define $B_{\rho}^{\tau}=\left\{\phi \in C_{[-\tau, 0]}:\|\phi\|_{r} \leq \rho\right\}$ as a closed ball of radius $\rho>0$ in $C_{[-\tau, 0]}$.

Definition 2. [15] The function $g: C_{[-\tau, 0]} \rightarrow \mathbb{R}$ is called $r$-homogeneous $\left(r_{i}>0, i=\overline{1, n}\right)$, if for any $\phi \in C_{[-\tau, 0]}$ the relation

$$
g\left(\Lambda_{r}(\lambda) \phi\right)=\lambda^{d} g(\phi)
$$

holds for some $d \in \mathbb{R}$ and all $\lambda>0$.

The function $f: C_{[-\tau, 0]} \rightarrow \mathbb{R}^{n}$ is called $r$-homogeneous $\left(r_{i}>0, i=\overline{1, n}\right)$, if for any $\phi \in C_{[-\tau, 0]}$ the relation

$$
f\left(\Lambda_{r}(\lambda) \phi\right)=\lambda^{d} \Lambda_{r}(\lambda) f(\phi)
$$

holds for some $d \geq-\min _{1 \leq i \leq n} r_{i}$ and all $\lambda>0$.

In both cases, the constant $d$ is called the degree of homogeneity.

The introduced notion of weighted homogeneity in $C_{[-\tau, 0]}$ is reduced to the standard one in $\mathbb{R}^{n}$ if $\tau \rightarrow 0$. An advantage of homogeneous systems described by ordinary differential equations is that any of its solutions can be obtained from another solution under the dilation rescaling and a suitable time re-parametrization. A similar property holds for some functional homogeneous systems.

Proposition 1. [16] Let $x: \mathbb{R}_{+} \rightarrow \mathbb{R}^{n}$ be a solution of the $r$-homogeneous system (1) with the degree $d=0$ for an initial condition $x_{0} \in C_{[-\tau, 0]}$. For any $\lambda>0$ define $y(t)=\Lambda_{r}(\lambda) x(t)$ for all $t \geq 0$, then $y(t)$ is also a solution of (1) with the initial condition $y_{0}=\Lambda_{r}(\lambda) x_{0}$.

\section{MAIN RESULTS}

This section has two parts. First, an extension of Proposition 1 is presented, and some useful tools for IOD stability analysis of nonlinear time-delay systems are introduced. Second, the links with finite-time stability are investigated using the homogeneity arguments.

\section{A. Scaling solutions of homogeneous systems with non-zero degree}

Proposition 1 establishes that all solutions of homogeneous time-delay systems of degree zero are interrelated, thus the local behavior implies global one. Some more complex relations can also be established for the case when the degree is not zero. 
Proposition 2. Let $x\left(t, x_{0}\right)$ be a solution of the $r$ homogeneous system (1) with the degree $d \neq 0$ for an initial condition $x_{0} \in C_{[-\tau, 0]}, \tau \in(0,+\infty)$. For any $\lambda>0$ the functional differential equation

$$
d y(t) / d t=f\left(y_{t}\right), t \geq 0
$$

with $y_{t} \in C_{\left[-\lambda^{-d} \tau, 0\right]}$, has a solution $y\left(t, y_{0}\right)=$ $\Lambda_{r}(\lambda) x\left(\lambda^{d} t, x_{0}\right)^{1}$ for all $t \geq 0$ with the initial condition $y_{0} \in C_{\left[-\lambda^{-d} \tau, 0\right]}, y_{0}(s)=\Lambda_{r}(\lambda) x_{0}\left(\lambda^{d} s\right)$ for $s \in\left[-\lambda^{-d} \tau, 0\right]$.

Proof. By definition $x_{\lambda^{d} t}(s)=x\left(\lambda^{d} t+s\right) \in \mathbb{R}^{n}$ and $y_{t}(s)=$ $\Lambda_{r}(\lambda) x_{\lambda^{d} t}(s)$ for any $-\lambda^{-d} \tau \leq s \leq 0$, then

$$
\begin{aligned}
\dot{y}(t) & =\frac{d}{d t}\left(\Lambda_{r}(\lambda) x\left(\lambda^{d} t\right)\right)=\lambda^{d} \Lambda_{r}(\lambda) f\left(x_{\lambda^{d} t}\right) \\
& =f\left(\Lambda_{r}(\lambda) x_{\lambda^{d} t}\right)=f\left(y_{t}\right)
\end{aligned}
$$

and $y(t)$ is a solution of (3) (dilation scales the argument of $f$ as $x(\theta) \rightarrow y\left(\lambda^{-d} \theta\right)$ for $\left.\theta \in[-\tau, 0]\right)$.

In [16], using Proposition 1 it has been shown for $d=$ 0 that local asymptotic stability implies global one (for the ordinary differential equations even more stronger conclusion can be obtained: local attractiveness implies global asymptotic stability [1]). In the present setting that result has the following correspondence.

Lemma 1. Let the system (1) be r-homogeneous with degree $d \neq 0$ and globally asymptotically stable at the origin for some delay $0<\tau_{0}<+\infty$, then it is globally asymptotically stable at the origin for any delay $0<\tau<+\infty$ (i.e. IOD).

Proof. Due to imposed hypothesis, for the delay $\tau_{0}>0$ for all $x_{0} \in C_{\left[-\tau_{0}, 0\right]}$ there is a function $\sigma \in \mathcal{K}$ such that $\left|x\left(t, x_{0}\right)\right|_{r} \leq$ $\sigma\left(\left\|x_{0}\right\|_{r}\right)$ for all $t \geq 0$ and $\lim _{t \rightarrow+\infty}\left|x\left(t, x_{0}\right)\right|_{r}=0$. Take some $\tau \in(0,+\infty)$ and select an initial condition $y_{0} \in C_{[-\tau, 0]}$, then for $\lambda=\left(\frac{\tau_{0}}{\tau}\right)^{1 / d}$ (this $\lambda$ is well defined since $d \neq 0)$ there exists $x_{0} \in C_{\left[-\tau_{0}, 0\right]}$ such that $y_{0}(s)=$ $\Lambda_{r}(\lambda) x_{0}\left(\lambda^{d} s\right)$ for $s \in[-\tau, 0]$, and $y\left(t, y_{0}\right)=\Lambda_{r}(\lambda) x\left(\lambda^{d} t, x_{0}\right)$ for all $t \geq 0$ by Proposition 2. Thus $\lim _{t \rightarrow+\infty}\left|y\left(t, y_{0}\right)\right|_{r}=$ $\lambda \lim _{t \rightarrow+\infty}\left|x\left(\lambda^{d} t, x_{0}\right)\right|_{r}=\lambda \lim _{t \rightarrow+\infty}\left|x\left(t, x_{0}\right)\right|_{r}=0$ and the solution $y\left(t, y_{0}\right)$ is converging asymptotically to the origin. In addition, $\left|y\left(t, y_{0}\right)\right|_{r}=\lambda\left|x\left(\lambda^{d} t, x_{0}\right)\right|_{r} \leq \lambda \sigma\left(\left\|x_{0}\right\|_{r}\right)=$ $\lambda \sigma\left(\lambda^{-1}\left\|y_{0}\right\|_{r}\right)$ for all $t \geq 0$, which implies stability of the system (1) for the delay $\tau$. The proven convergence to the origin and stability give the global asymptotic stability of the system for an arbitrary delay $\tau \in(0,+\infty)$.

It is well known fact for linear systems (homogeneous systems of degree $d=0$ ) that its stability for a sufficiently small delay does not imply stability for all $\tau \in(0,+\infty)$. For nonlinear homogeneous systems with degree $d \neq 0$ this is not the case, according to the result of Lemma 1 if they are globally stable for some delay, they preserve their stability for an arbitrary delay $\tau \in(0,+\infty)$. This is a surprising advantage of nonlinear time-delay systems.

Further let us consider several useful consequences of Proposition 2 and Lemma 1.

\footnotetext{
${ }^{1}$ If time is scaled $t \rightarrow \lambda^{d} t$ then the argument of $f: C_{[-\tau, 0]} \rightarrow \mathbb{R}^{n}$ in (1) is also scaled to $\left.f: C_{\left[-\lambda^{-d}\right.} \tau, 0\right] \rightarrow \mathbb{R}^{n}$ in (3).
}

Corollary 1. Let the system (1) be r-homogeneous with degree $d \neq 0$ and asymptotically stable at the origin into the set $\Omega=B_{\rho}^{\tau}=\left\{\phi \in C_{[-\tau, 0]}:\|\phi\|_{r} \leq \rho\right\}$ for some $0<\rho<+\infty$ for any value of delay $0 \leq \tau<+\infty$, then it is globally asymptotically stable at the origin IOD.

Proof. For any $\tau>0$ take $y_{0} \in C_{[-\tau, 0]}, y_{0} \notin B_{\rho}^{\tau}$, then according to Proposition 2 there is $0<\lambda<+\infty(\lambda=$ $\left.\rho^{-1}\left\|y_{0}\right\|_{r}\right)$ and $x_{0} \in B_{\rho}^{\lambda^{d} \tau}$ such that $y_{0}(s)=\Lambda_{r}(\lambda) x_{0}\left(\lambda^{d} s\right)$ for $s \in[-\tau, 0]$ and $y\left(t, y_{0}\right)=\Lambda_{r}(\lambda) x\left(\lambda^{d} t, x_{0}\right)$ for all $t \geq 0$. Since $x(t)$ converges asymptotically to the origin, the same property is satisfied for $y(t)$ and it enters the set $B_{\rho}^{\tau}$ in a finite time.

Corollary 2. Let the system (1) be r-homogeneous with degree $d<0$ and asymptotically stable at the origin into the set $B_{\rho^{\prime}}^{\tau}$ for some $0<\rho^{\prime}<+\infty$ for any value of delay $0 \leq \tau \leq \tau_{0}$ with $0<\tau_{0}<+\infty$, then it is globally asymptotically stable at the origin IOD.

Proof. Setup $\rho<\rho^{\prime}$, take $y_{0} \in C_{\left[-\tau_{0}, 0\right]}, y_{0} \notin B_{\rho}^{\tau_{0}}$ and select $1<\lambda=\rho^{-1}\left\|y_{0}\right\|_{r}$ for some $x_{0} \in B_{\rho}^{\lambda^{d} \tau_{0}},\left\|x_{0}\right\|_{r}=\rho$ such that $y_{0}(s)=\Lambda_{r}(\lambda) x_{0}\left(\lambda^{d} s\right)$ for $s \in\left[-\tau_{0}, 0\right]$ (note that $\tau=\lambda^{d} \tau_{0} \leq \tau_{0}$ since $d<0$ and $\lambda>1$ ), then according to Proposition $2 y\left(t, y_{0}\right)=\Lambda_{r}(\lambda) x\left(\lambda^{d} t, x_{0}\right)$ for all $t \geq 0$. Since $x(t)$ converges asymptotically to the origin, the same property is satisfied for $y(t)$. To prove stability note that there is a function $\sigma_{\tau} \in \mathcal{K}$ such that $\left|x\left(t, x_{0}\right)\right|_{r} \leq \sigma_{\tau}\left(\left\|x_{0}\right\|_{r}\right)$ for all $t \geq 0$ and $x_{0} \in B_{\rho}^{\tau}$ with $0 \leq \tau \leq \tau_{0}$, define $R=\sup _{0 \leq \tau \leq \tau_{0}} \sigma_{\tau}(\rho)<+\infty\left(R\right.$ is finite since $\left.\rho<\rho^{\prime}\right)$ then $\left|y\left(t, y_{0}\right)\right|_{r}=\lambda\left|x\left(\lambda^{d} t, x_{0}\right)\right|_{r} \leq \lambda R=\rho^{-1} R\left\|y_{0}\right\|_{r}$ for all $t \geq 0$ and $y_{0} \in C_{\left[-\tau_{0}, 0\right]}$. Therefore, the system is globally asymptotically stable for the delay $\tau_{0}$. Using the result of Lemma 1 the claim about uniformity of global asymptotic stability with respect to the delay can be recovered.

Corollary 3. Let the system (1) be r-homogeneous with degree $d>0$ and the set $B_{\rho}^{\tau}$ for some $0<\rho<+\infty$ be uniformly globally asymptotically stable for any value of delay $0 \leq \tau \leq$ $\tau_{0}, 0<\tau_{0}<+\infty^{2}$, then (1) is globally asymptotically stable at the origin IOD.

Proof. Take $\rho^{\prime}>\rho$ and $\lambda=\frac{\left\|y_{0}\right\|_{r}}{\rho^{\prime}} \leq 1$ for any $y_{0} \in B_{\rho}^{\tau_{0}}$ then there exists $x_{0} \in B_{\rho^{\prime}}^{\lambda^{d}} \tau_{0},\left\|x_{0}\right\|_{r}=\rho^{\prime}$ such that $y_{0}(s)=\Lambda_{r}(\lambda) x_{0}\left(\lambda^{d} s\right)$ for $s \in\left[-\tau_{0}, 0\right]$. Note that $\lambda^{d} \tau_{0} \leq \tau_{0}$ for $\lambda \leq 1$ and $d>0$. According to Proposition $2 y\left(t, y_{0}\right)=\Lambda_{r}(\lambda) x\left(\lambda^{d} t, x_{0}\right)$ for all $t \geq 0$. Since $\left|x\left(t, x_{0}\right)\right|_{r} \leq \sigma_{\lambda^{d} \tau_{0}}\left(\left\|x_{0}\right\|_{r}\right)$ for all $t \geq 0$, then $\left|y\left(t, y_{0}\right)\right|_{r} \leq \rho^{\prime-1}\left\|y_{0}\right\|_{r} \sigma_{\lambda^{d} \tau_{0}}\left(\rho^{\prime}\right)$ for all $t \geq 0$ and all $y_{0} \in B_{\rho}^{\tau_{0}}$. Define $R=\sup _{\tau \in\left[0, \tau_{0}\right]} \sigma_{\tau}\left(\rho^{\prime}\right)$, then $\left|y\left(t, y_{0}\right)\right|_{r} \leq$ $\eta\left(\left\|y_{0}\right\|_{r}\right)=\max \left\{\rho^{\prime-1}\left\|y_{0}\right\|_{r} R, \sigma_{\tau_{0}}\left(\left\|y_{0}\right\|_{r}\right)\right\}$ for all $t \geq 0$ for any $y_{0} \in C_{\left[-\tau_{0}, 0\right]}$ and the system (1) is globally stable for the delay $\tau_{0}$ (in this case $\eta(s) \geq s$ for all $s \geq 0$ ). Select $\varepsilon>0$ (without loosing generality assume that $\eta^{-1}(\epsilon)<\rho$ ) and take $\lambda=\frac{\eta^{-1}(\varepsilon)}{\rho+\varepsilon} \leq \frac{\varepsilon}{\rho+\varepsilon}<1$, then for any $y_{0} \in B_{\rho}^{\tau_{0}} \backslash B_{\eta^{-1}(\varepsilon)}^{\tau_{0}}$

${ }^{2}$ In this case for any $0 \leq \tau \leq \tau_{0}$, any $\varepsilon>0$ and $\kappa \geq 0$ there is $0 \leq T_{\kappa, \tau}^{\varepsilon}<+\infty$ such that $\left\|x_{t, x_{0}}\right\|_{r} \leq \rho+\varepsilon$ for all $t \geq \bar{T}_{\kappa, \tau}^{\varepsilon}$ for any $x_{0} \in B_{\kappa}^{\tau}$, and $\left|x\left(t, x_{0}\right)\right|_{r} \leq \sigma_{\tau}\left(\left\|x_{0}\right\|_{r}\right)$ for all $t \geq 0$ for some function $\sigma_{\tau} \in \mathcal{K}_{\infty}$ for all $x_{0} \notin B_{\rho}^{\tau}$. 
there exists $x_{0} \in B_{\lambda^{-1} \rho}^{\lambda^{d} \tau_{0}}$ such that $y_{0}(s)=\Lambda_{r}(\lambda) x_{0}\left(\lambda^{d} s\right)$ for $s \in\left[-\tau_{0}, 0\right]$. Since again $y\left(t, y_{0}\right)=\Lambda_{r}(\lambda) x\left(\lambda^{d} t, x_{0}\right)$ for all $t \geq 0$ and $\left\|x_{t, x_{0}}\right\|_{r} \leq \rho+\varepsilon$ for all $t \geq T_{\lambda^{-1} \rho, \lambda^{d} \tau_{0}}^{\varepsilon}$, then $\left\|y_{t, y_{0}}\right\|_{r} \leq \eta^{-1}(\varepsilon) \leq \varepsilon$ for all $t \geq \lambda^{-d} T_{\lambda^{-1} \rho, \lambda^{d} \tau_{0}}^{\varepsilon}$. For $y_{0} \in B_{\eta^{-1}(\varepsilon)}^{\tau_{0}}$ we have $\left\|y_{t, y_{0}}\right\|_{r} \leq \varepsilon$ for all $t \geq 0$, that implies uniform attractivity of the origin for (1) with the delay $\tau_{0}$. From these properties the global asymptotic stability for the delay $\tau_{0}$ is deduced. Finally, the claim of the corollary for any delay $\tau \in(0,+\infty)$ follows the conclusion of Lemma 1.

Remark 1. The requirements of corollaries 2 and 3 can be relaxed asking stability of $B_{\rho}^{\tau_{0}}$ for some $0<\rho<+\infty$ for a fixed $0<\tau_{0}<+\infty$ and for $\tau_{0}=0$, then the corresponding stability of $B_{\rho}^{\tau}$ for all $0 \leq \tau \leq \tau_{0}$ can be obtained using the arguments of Lemma 1.

In addition, due to ISS property well inherited by homogeneous systems (see [30], [16] for details), under mild conditions its stability for $\tau=0$ implies the same property for sufficiently small delay. Similar results, connecting ISS and stability of the systems with respect to time delays, have been established in [31], [32].

Lemma 2. Let $f\left(x_{t}\right)=F[x(t), x(t-\tau)]$ in (1) and the system (1) be r-homogeneous with degree $d>0$ and globally asymptotically stable at the origin for $\tau=0$, then for any $\rho>0$ there is $0<\tau_{0}<+\infty$ such that (1) is asymptotically stable at the origin into $B_{\rho}^{\tau}$ for any delay $0 \leq \tau \leq \tau_{0}$.

Proof. For some $\rho>0$ and $\tau>0$ let us consider $x_{0} \in B_{\rho}^{\tau}$, then

$$
x\left(t, x_{0}\right)=x_{0}(0)+\int_{0}^{t} F\left[x\left(s, x_{0}\right), x\left(s-\tau, x_{0}\right)\right] d s
$$

is the unique solution of (1) if it is defined on the interval $[0, t]$ for $t \geq 0$ [11], $\left\|x_{0}\right\| \leq \bar{\rho}_{r}(\rho)$ by (2). Since $F$ is locally Lipschitz, then for all $\|\phi\| \leq 2 \bar{\rho}_{r}(\rho)$ there exists $k>0$ such that

$$
|F[\phi(0), \phi(-\tau)]| \leq k\|\phi\| .
$$

Select $\tau>0$ such that $k \tau<0.5$, then assuming that $\left\|x_{s}\right\| \leq$ $2 \bar{\rho}_{r}(\rho)$ for all $s \in[0, t)$ we obtain

$$
\begin{aligned}
\left|x\left(t, x_{0}\right)\right| & \leq\left|x_{0}(0)\right|+\int_{0}^{t}\left|F\left[x\left(s, x_{0}\right), x\left(s-\tau, x_{0}\right)\right]\right| d s \\
& \leq\left|x_{0}(0)\right|+k \int_{0}^{t}\left\|x_{s}\right\| d s \\
& \leq\left|x_{0}(0)\right|+k t \sup _{0 \leq s<t}\left\|x_{s}\right\| \leq(1+2 k t) \bar{\rho}_{r}(\rho)
\end{aligned}
$$

and $\left|x\left(t, x_{0}\right)\right| \leq 2 \bar{\rho}_{r}(\rho)$ for $0 \leq t \leq \tau$, thus $\left\|x_{s}\right\| \leq 2 \bar{\rho}_{r}(\rho)$ for $s \in[0, \tau]$. Therefore, for any $\rho>0$ there exists $\tau>0$ such that for $x_{0} \in B_{\rho}^{\tau}$ we have $x_{t} \in B_{\rho^{\prime}}^{\tau}$ for $t \in[0, \tau]$ and $\rho^{\prime}=\rho_{r}^{-1}\left[2 \bar{\rho}_{r}(\rho)\right]$ by $(2)$.

The conditions of this lemma means that $F\left[\Lambda_{r}(\lambda) x, \Lambda_{r}(\lambda) z\right]=\lambda^{d} \Lambda_{r}(\lambda) F[x, z]$ for any $x, z \in \mathbb{R}^{n}$ and $\lambda \in(0,+\infty)$. In addition, $x=0$ for the system

$$
\dot{x}=F(x, x)
$$

is globally asymptotically stable. Note that the system (4) is also homogeneous of degree $d>0\left(F\left[\Lambda_{r}(\lambda) x, \Lambda_{r}(\lambda) x\right]=\right.$ $\lambda^{d} \Lambda_{r}(\lambda) F[x, x]$ for any $x \in \mathbb{R}^{n}$ and $\left.\lambda \in(0,+\infty)\right)$, then according to [4], [5] there is a differentiable and $r$-homogeneous Lyapunov function $V: \mathbb{R}^{n} \rightarrow \mathbb{R}_{+}$of degree $v>0$ such that

$$
\begin{gathered}
a=-\sup _{\xi \in \mathbb{S}_{r}} D^{+} V(\xi) F(\xi, \xi)>0, \\
0<b=\sup _{|\xi|_{r} \leq 1}\left|\frac{\partial V(\xi)}{\partial \xi}\right|<+\infty, \\
c_{1}=\inf _{\xi \in \mathbb{S}_{r}} V(\xi), c_{2}=\sup _{\xi \in \mathbb{S}_{r}} V(\xi), \\
c_{1}|x|_{r}^{v} \leq V(x) \leq c_{2}|x|_{r}^{v} \quad \forall x \in \mathbb{R}^{n} .
\end{gathered}
$$

Consider derivative of this function calculated for the system (1) for some $\phi \in C_{[-2 \tau, 0]}$. To this end define $\lambda_{1}=|\phi(0)|_{r}$ and $\lambda_{2}=\|\phi\|_{r}$ with $\phi(0)=\Lambda_{r}\left(\lambda_{1}\right) \xi$ for some $\xi \in \mathbb{S}_{r}$ and $\phi=\Lambda_{r}\left(\lambda_{2}\right) \varphi$ for some $\varphi \in \mathcal{S}_{r} \subset C_{[-2 \tau, 0]}$ respectively, $\lambda_{2} \geq$ $\lambda_{1}$ by definition of the norms and $|\varphi(0)|_{r} \leq|\xi|_{r}=1$ (a necessity to define two dilation transformations with $\lambda_{1}$ and $\lambda_{2}$ is originated by the fact that the state vectors of (1) and (4) belong to different spaces, $\mathbb{R}^{n}$ and $C_{[-\tau, 0]}$ ):

$$
\begin{gathered}
D^{+} V(\phi(0)) F[\phi(0), \phi(-\tau)]=D^{+} V(\phi(0)) F[\phi(0), \phi(0)] \\
+D^{+} V(\phi(0))\{F[\phi(0), \phi(-\tau)]-F[\phi(0), \phi(0)]\} \\
=\lambda_{1}^{d+v} D^{+} V(\xi) F[\xi, \xi]+\lambda_{2}^{d+v} D^{+} V(\varphi(0))\{F[\varphi(0), \varphi(-\tau)] \\
-F[\varphi(0), \varphi(0)]\} .
\end{gathered}
$$

Since $d>0$ and the function $F$ is locally Lipschitz with respect to both arguments, then for $\varphi \in \mathcal{S}_{r}$ there exists $L>0$ such that

$$
\mid F[\varphi(0), \varphi(-\tau)]-F[\varphi(0), \varphi(0)|\leq L| \varphi(0)-\varphi(-\tau) \mid .
$$

Let us consider the solutions of (1) for $t \geq \tau$ (we have shown above that for $x_{0} \in B_{\rho}^{\tau}$ we have $x_{t} \in B_{\rho^{\prime}}^{\tau}$ for $t \in[0, \tau]$ for any $\rho>0, \rho^{\prime}=\rho_{r}^{-1}\left[2 \bar{\rho}_{r}(\rho)\right]$ and a properly selected $\tau<0.5 / k)$, then there exists $M>0$ such that $\mid \varphi(0)-$ $\varphi(-\tau) \mid \leq M \tau$, where $M=\sup _{|z|_{r} \leq \rho^{\prime},|y|_{R} \leq \rho^{\prime}}|F[z, y]|$. In order to prove asymptotic stability, the Lyapunov-Razumikhin approach will be applied [10]. To this end, assume for some $\gamma>1$ that $c_{1} \sup _{\theta \in[-2 \tau, 0]}|\phi(\theta)|_{r}^{v} \leq \sup _{\theta \in[-2 \tau, 0]} V[\phi(\theta)]<$ $\gamma V[\phi(0)] \leq \gamma c_{2}|\phi(0)|_{r}^{v}$, therefore $\sup _{\theta \in[-2 \tau, 0]}|\phi(\theta)|_{r} \leq$ $\left(\gamma c_{1}^{-1} c_{2}\right)^{1 / v}|\phi(0)|_{r}=\left(\gamma c_{1}^{-1} c_{2}\right)^{1 / v} \lambda_{1}$, then

$$
\begin{aligned}
\lambda_{2} & =\|\phi\|_{r}=\left(\sum_{i=1}^{n} \sup _{\theta \in[-2 \tau, 0]}\left|\phi_{i}(\theta)\right|^{\rho / r_{i}}\right)^{1 / \rho} \\
& \leq\left(\sum_{i=1}^{n} \sup _{\theta \in[-2 \tau, 0]}|\phi(\theta)|_{r}^{\rho}\right)^{1 / \rho} \\
& =n^{1 / \rho} \sup _{\theta \in[-2 \tau, 0]}|\phi(\theta)|_{r} \leq R \lambda_{1},
\end{aligned}
$$

where $R=n^{1 / \rho}\left(\gamma c_{1}^{-1} c_{2}\right)^{1 / v}$. Finally,

$$
\begin{aligned}
D^{+} V(\phi(0)) F[\phi(0), \phi(-\tau)] & \leq \tau b L M \lambda_{2}^{d+v}-a R^{-d-v} \lambda_{2}^{d+v} \\
& \leq\|\phi\|_{r}^{d+v}\left(\tau b L M-a R^{-d-v}\right),
\end{aligned}
$$

and for all $0 \leq \tau \leq \tau_{0}=\min \left\{0.5 / k, \frac{a R^{-d-v}-\epsilon}{b L M}\right\}$ for some $\epsilon \in\left(0, a R^{-d-v}\right)$ we have

$\max _{\theta \in[-2 \tau, 0]} V[\phi(\theta)]<\gamma V[\phi(0)] \Rightarrow D^{+} V(\phi(0)) F[\phi(0), \phi(-\tau)] \leq-\epsilon|\phi(0)|_{r}^{d+v}$

for all $\phi \in C_{[-2 \tau, 0]}$ that are solutions of (1) for $t \geq \tau$. Consequently [10], the trajectories of (1) are strictly decreasing 
to zero and stay bounded for $t \geq \tau$, but they also stay bounded for $0 \leq t \leq \tau$ with $\left\|x_{0}\right\|_{r} \leq \rho$, which gives the desired result.

The results presented in this section open the door for a wealthy stability analysis of nonlinear time-delay systems.

\section{B. Finite-time stability}

Assume that the system (1) is finite-time stable for some $\tau>0$ and the settling-time functional is continuous and $T_{0}(0)=0$. According to such a definition there is $x_{0} \in C_{[-\tau, 0]}$ such that $T_{0}\left(x_{0}\right) \leq \tau$ and at the instant $T_{0}\left(x_{0}\right)$ the righthand side is still dependent on initial conditions. Thus without additional assumptions on the right-hand side $f\left(x_{\tau}\right)$ and its dependence on $x_{\tau}(0)$, or without skipping the continuity requirement of $T_{0}\left(x_{0}\right)$, an existence of finite-time stability phenomenon for time-delay systems is questionable [29].

For the ordinary differential equations, finite-time stability is frequently related with homogeneity. It is well known that a homogeneous continuous-time system of negative degree is finite-time stable iff it has the origin locally attracting [2]. The recent results for finite-time stability analysis of timedelay systems [27], [28], [29] are not based on homogeneity. The paper [27] proposes design of a control, which implicitly contains some prediction mechanisms and time-varying gains in order to compensate the delay influence on the system dynamics and guarantee a kind of finite-time stability for the closed-loop system. The main result of [28] is required to find a Lyapunov-Krasovskii functional $V(\phi)$ whose derivative is upper bounded by a certain negative function of the functional $V(\phi)$ itself (it is more restricted condition than in conventional Lyapunov-Krasovskii approach, where a function of $|\phi(0)|$ is required). Using the result of Proposition 2 on rescaling of solutions of homogeneous systems with non-zero degree, the following conditions of finite-time stability in time-delay systems can be established.

Theorem 1. Let the system (1) be r-homogeneous with degree $d<0$ and globally asymptotically stable at the origin IOD. Let $0<\mathcal{T}<+\infty$, where $\mathcal{T}$ is defined in one of the following ways for $q \in(0,1)$ :

(a) $\mathcal{T}=\sup _{\tau \in(0,+\infty)} \sup _{x_{0} \in C_{[-\tau, 0]},\left\|x_{0}\right\|_{r}=1} T_{x_{0}}$ and $T_{x_{0}}$ is such that $\left\|x_{t, x_{0}}\right\|_{r} \leq q\left\|x_{0}\right\|_{r}$ for all $t \geq T_{x_{0}}$;

(b) $\mathcal{T}=\sup _{\tau \in(0,+\infty)} \sup _{x_{0} \in C_{[-\tau, 0]},\left\|x_{0}\right\|_{r} \geq 1} T_{x_{0}}$ and $T_{x_{0}}$ is such that $\left|x\left(t, x_{0}\right)\right|_{r} \leq q\left|x_{0}(0)\right|_{r}$ for all $t \geq T_{x_{0}}$.

Then (1) is finite-time stable at the origin IOD.

Proof. Since the system is globally asymptotically stable independently of the delay, then it is stable and according to definition of finite-time stability it is required to show finite-time convergence to the origin for all initial conditions and delays. Select some $\tau \in(0,+\infty)$. Since the system is globally asymptotically stable, for any $z_{0} \in C_{[-\tau, 0]}$ there is $0 \leq t_{z_{0}}<+\infty$ such that $\left\|x\left(t+\cdot, z_{0}\right)\right\|_{r} \leq 1$ for all $t \geq t_{z_{0}}$. Next, the time of convergence to the origin equals $t_{z_{0}}+T_{1, z_{0}}$, where $T_{1, z_{0}}$ is the time of convergence from the sphere $\mathcal{S}_{r}$ to the origin. It is necessary to show that $T_{1, x_{0}}$ is finite for any $x_{0} \in \mathcal{S}_{r}$.
First, consider the part $(a)$. By conditions of the theorem, $\left\|x_{t, x_{0}}\right\|_{r} \leq q\left\|x_{0}\right\|_{r}$ for any $x_{0} \in C_{[-\tau, 0]}, \quad\left\|x_{0}\right\|_{r}=1$ for all $t \geq \mathcal{T} \geq T_{x_{0}}$ and any $\tau \in(0,+\infty)$. Take $y_{0} \in C_{[-\tau, 0]}, \quad\left\|y_{0}\right\|_{r}=q^{k}$ for some integer $k \geq 1$. Then there exists $x_{0} \in C_{\left[-q^{k d} \tau, 0\right]},\left\|x_{0}\right\|_{r}=1$ such that $y_{0}(s)=\Lambda_{r}\left(q^{k}\right) x_{0}\left(q^{k d} s\right)$ for $s \in[-\tau, 0]$ and according to Proposition $2 y\left(t, y_{0}\right)=\Lambda_{r}\left(q^{k}\right) x\left(q^{k d} t, x_{0}\right)$ for all $t \geq 0$. Therefore, $\left\|y_{t, y_{0}}\right\|_{r}=q^{k}\left\|x_{q^{k d} t, x_{0}}\right\|_{r} \leq q q^{k}\left\|x_{0}\right\|_{r}=q\left\|y_{0}\right\|_{r}$ for all $t \geq T_{y_{0}}=q^{-k d} T_{x_{0}}$. Thus $\left\|y_{t, y_{0}}\right\|_{r} \leq q\left\|y_{0}\right\|_{r}$ for any $y_{0} \in C_{[-\tau, 0]},\left\|y_{0}\right\|_{r}=q^{k}$ for all $t \geq q^{-k d} \mathcal{T} \geq T_{y_{0}}$. Finally,

$$
\begin{gathered}
T_{1, x_{0}}=\left.T_{x_{0}}\right|_{\left\|x_{0}\right\|_{r}=1}+\left.T_{y_{0}}\right|_{\left\|y_{0}\right\|_{r}=q}+\left.T_{y_{0}}\right|_{\left\|y_{0}\right\|_{r}=q^{2}}+\ldots \\
=\mathcal{T}+q^{-d} \mathcal{T}+q^{-2 d} \mathcal{T}+\cdots=\mathcal{T} \sum_{k \geq 1} q^{-k d}=\frac{\mathcal{T}}{1-q^{-d}}<+\infty .
\end{gathered}
$$

This consideration can be repeated for any $\tau \in(0,+\infty)$.

For the part $(b)$ the proof follows exactly the same arguments (it is necessary to take $y_{0} \in C_{[-\tau, 0]},\left|y_{0}(0)\right|_{r}=q^{k}$ for some integer $k \geq 1$. Then there exists $x_{0} \in C_{\left[-q^{k d} \tau, 0\right]}, 1 \leq$ $\left\|x_{0}\right\|_{r}$ such that $y_{0}(s)=\Lambda_{r}\left(q^{k}\right) x_{0}\left(q^{k d} s\right)$ for $s \in[-\tau, 0]$ and according to Proposition $2 y\left(t, y_{0}\right)=\Lambda_{r}\left(q^{k}\right) x\left(q^{k d} t, x_{0}\right)$ for all $t \geq 0$, and $\left|y\left(t, y_{0}\right)\right|_{r} \leq q\left|y_{0}(0)\right|_{r}$ for all $t \geq q^{-k d} \mathcal{T} \geq$ $\left.T_{y_{0}}\right)$.

Lemma 1 and Corollary 2 can be used to check the global asymptotic stability IOD property that is required in Theorem 1. Uniformity of $\mathcal{T}$ with respect to delay value implies that finite-time convergence in (1) is possible under a "weak" dependence on delay. The main result of this theorem is another demonstration for complexity of existence of finitetime stability in the functional differential equations [29]. Of course these results are obtained for homogeneous systems only, but they provide a good intuition about other cases also.

\section{Application to StABilization OF A CHAin OF INTEGRATORS}

In [2], for a chain of integrators

$$
\begin{aligned}
\dot{\xi}_{i} & =\xi_{i+1}, i=1, \ldots, n-1, \\
\dot{\xi}_{n} & =u
\end{aligned}
$$

where $x=\left(\xi_{1}, \ldots, \xi_{n}\right)^{T} \in \mathbb{R}^{n}$ is the state and $u \in \mathbb{R}$ is the control, the following stabilizing controller has been proposed:

$$
u=-\sum_{i=1}^{n} k_{i}\left|\xi_{i}\right|^{\alpha_{i}} \operatorname{sign}\left(\xi_{i}\right),
$$

where $k_{i}$ form a Hurwitz polynomial and $\alpha_{i} \in \mathbb{R}_{+}$. If the powers $\alpha_{i}$ are selected in a way providing homogeneity of the closed-loop system and if all of them are smaller and sufficiently close to 1 , then the system is finite-time stable [2]. For the case $n=2, \alpha_{1}=\frac{\alpha}{2-\alpha}, \alpha_{2}=\alpha$ and an arbitrary value of $\alpha \in(0,1)$ a solution has been given in [33]. Let us assume that the state is available for measurements with delays $\tau_{i} \in\left(0, \tau_{\max }\right), 0<\tau_{\max }<+\infty$ :

$$
u^{\prime}(t)=-\sum_{i=1}^{n} k_{i}\left|\xi_{i}\left(t-\tau_{i}\right)\right|^{\alpha_{i}} \operatorname{sign}\left[\xi_{i}\left(t-\tau_{i}\right)\right],
$$

then applying the result of Lemma 2 (which can be easily extended to the case of multiple delays) to the closed-loop 


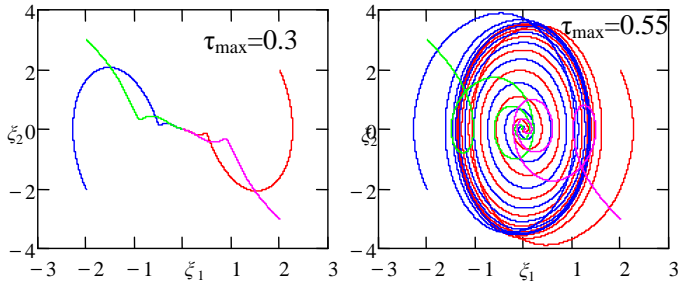

Figure 1. Trajectories of stabilized delayed double integrator

system we obtain that it is locally asymptotically stable in $C_{\left[-\tau_{\max }, 0\right]}$ if $\alpha_{i}>1$ and sufficiently close to 1 . Indeed, in this case the homogeneity degree is positive (Corollary 2 cannot be applied) and we can use the same technique as in [2] to prove global asymptotic stability of the delayfree control (for the case $n=2$ the Lyapunov function $V(x)=\frac{k_{1}}{\alpha_{1}+1}\left|\xi_{1}\right|^{\alpha_{1}+1}+\frac{1}{2} \xi_{2}^{2}$ can be used). These results are related with ones obtained in [21] for a bounded global stabilizer of the chain of integrators, in both cases increasing of the delay leads to decreasing the set of attraction.

The results of computer simulation of this system for two different values of delays (for $n=2, k_{1}=1, k_{2}=2, \alpha_{1}=$ 1.5, $\alpha_{2}=1.2$ ) is shown in Fig. 1. As we can conclude, fixing the initial conditions and increasing the value of delay the system becomes more oscillatory and finally unstable for a sufficiently big value of $\tau_{\max }$.

\section{Conclusions}

Extensions of the homogeneity theory are obtained for timedelay systems. It is shown that for any degree of homogeneity the solutions of a homogeneous system are interrelated subject to the delay rescaling. Next, this fact is utilized in order to show that local uniform asymptotic stability of homogeneous systems implies global one, and that for nonlinear homogeneous systems with non-zero degree, global asymptotic stability for a delay endorses this property for an arbitrary large delay. Thus analysis of stability in nonlinear time-delay systems differs significantly from the linear case, but in a positive sense: being stable for a delay such systems save this property for any delay value, which is an interesting advantage of nonlinear time-delay systems. Efficiency of the proposed approach is demonstrated on application to a finitetime stabilizing control from [2].

\section{REFERENCES}

[1] A. Bacciotti and L. Rosier, Liapunov Functions and Stability in Control Theory, vol. 267 of Lecture Notes in Control and Inform. Sci. Berlin: Springer, 2001.

[2] S. Bhat and D. Bernstein, "Geometric homogeneity with applications to finite-time stability," Mathematics of Control, Signals and Systems, vol. 17, pp. 101-127, 2005.

[3] M. Kawski, Homogeneous feedback stabilization, vol. 7 of Progress in systems and control theory: New trends in systems theory. Birkhäuser, 1991.

[4] V. Zubov, "On systems of ordinary differential equations with generalized homogenous right-hand sides," Izvestia vuzov. Mathematica., vol. 1, pp. 80-88, 1958. in Russian.

[5] L. Rosier, "Homogeneous Lyapunov function for homogeneous continuous vector field," Systems\&Control Lett., vol. 19, pp. 467-473, 1992.

[6] D. Efimov and W. Perruquetti, "Oscillations conditions in homogenous systems," in Proc. NOLCOS'10, (Bologna), pp. 1379-1384, 2010.
[7] V. Andrieu, L. Praly, and A. Astolfi, "Homogeneous approximation, recursive observer design, and output feedback," SIAM J. Control Optimization, vol. 47, no. 4, pp. 1814-1850, 2008.

[8] J. Chiasson and J. Loiseau, eds., Applications of Time Delay Systems, vol. 352 of Lecture Notes in Control and Information Sciences. Springer, 2007.

[9] T. Erneux, Applied Delay Differential Equations. NY: Springer, 2009.

[10] K. Gu, K. Kharitonov, and J. Chen, Stability of Time-Delay Systems. Control Engineering, Boston: Birkhäuser, 2003.

[11] J. Hale, Theory of Functional Differential Equations. Springer-Verlag, 1977.

[12] V. Kolmanovsky and V. Nosov, Stability of functional differential equations. San Diego: CA: Academic, 1986.

[13] J.-P. Richard, "Time-delay systems: an overview of some recent advances and open problems," Automatica, vol. 39, pp. 1667-1694, 2003.

[14] E. Fridman, Introduction to Time-Delay Systems: Analysis and Control. Basel: Birkhäuser, 2014.

[15] D. Efimov and W. Perruquetti, "Homogeneity for time-delay systems," in Proc. IFAC WC 2011, (Milan), 2011.

[16] D. Efimov, W. Perruquetti, and J.-P. Richard, "Development of homogeneity concept for time-delay systems," SIAM J. Control Optim., vol. 52, no. 3, pp. 1403-1808, 2014.

[17] A. Aleksandrov and A. Zhabko, "On the asymptotic stability of solutions of nonlinear systems with delay," Siberian Mathematical Journal, vol. 53, no. 3, pp. 393-403, 2012.

[18] F. Asl and A. Ulsoy, "Analytical solution of a system of homogeneous delay differential equations via the Lambert function," in Proc. American Control Conference, (Chicago), pp. 2496-2500, 2000.

[19] V. Bokharaie, O. Mason, and M. Verwoerd, "D-stability and delayindependent stability of homogeneous cooperative systems," IEEE Trans. Automatic Control, vol. 55, no. 12, pp. 2882-2885, 2010.

[20] J. Diblik, "Asymptotic equilibrium for homogeneous delay linear differential equations with 1-perturbation term," Nonlinear Analysis, Theory, Methods \& Applicarions, vol. 30, no. 6, pp. 3927-3933, 1997.

[21] F. Mazenc, S. Mondie, and S.-I. Niculescu, "Global asymptotic stabilization for chains of integrators with a delay in the input," in Proc. 40th IEEE Conference on Decision and Control, vol. 2, pp. 1843-1848, 2001.

[22] E. Roxin, "On finite stability in control systems," Rendiconti del Circolo Matematico di Palermo, vol. 15, pp. 273-283, 1966.

[23] P. Dorato, "An overview of finite-time stability," in Current Trends in Nonlinear Systems and Control (L. Menini, L. Zaccarian, and C. T. Abdallah, eds.), Systems \& Control: Foundations \& Applications, pp. 185194, Boston: Birkhäuser, 2006.

[24] E. Moulay and W. Perruquetti, "Finite time stability and stabilization of a class of continuous systems," J. Mathematical Analysis Applications, vol. 323, no. 2, pp. 1430-1443, 2006.

[25] S. Nersesov, W. Haddad, and Q. Hui, "Finite-time stabilization of nonlinear dynamical systems via control vector lyapunov functions," $J$. Franklin Inst., vol. 345, pp. 819-837, 2008.

[26] A. Polyakov, "Nonlinear feedback design for fixed-time stabilization of linear control systems," Automatic Control, IEEE Transactions on, vol. 57, no. 8, pp. 2106-2110, 2012.

[27] I. Karafyllis, "Finite-time global stabilization by means of time-varying distributed delay feedback," SIAM J. Control Optimization, vol. 45, no. 1 , pp. 320-342, 2006.

[28] E. Moulay, M. Dambrine, N. Yeganefar, and W. Perruquetti, "Finite-time stability and stabilization of time-delay systems," Systems Control Lett., vol. 57, pp. 561-566, 2008.

[29] D. Efimov, A. Polyakov, E. Fridman, W. Perruquetti, and J.-P. Richard, "Comments on finite-time stability of time-delay systems," Automatica, vol. 50, no. 7, pp. 1944-1947, 2014.

[30] E. Bernuau, A. Polyakov, D. Efimov, and W. Perruquetti, "Verification of iss, iiss and ioss properties applying weighted homogeneity," Systems \& Control Letters, vol. 62, no. 12, pp. 1159-1167, 2013.

[31] F. Mazenc, M. Malisoff, and T. N. Dinh, "Robustness of nonlinear systems with respect to delay and sampling of the controls," Automatica, vol. 49, no. 6, pp. 1925-1931, 2013.

[32] A. Teel, D. Nesic, and P. Kokotovic, "A note on input-to-state stability of sampled-data nonlinear systems," in Proc. 37th IEEE Conference on Decision and Control, vol. 3, pp. 2473-2478, 1998.

[33] E. Bernuau, W. Perruquetti, D. Efimov, and E. Moulay, "Finite time output stabilization of the double integrator," in 51st IEEE Conference on Decision and Control, (Hawaii, USA), pp. 5906-5911, 2012. 